\title{
Porous Au-Ag Nanospheres with High-Density and Highly Accessible Hotspots for SERS Analysis
}

Kai Liu, ${ }^{\dagger}$ Yaocai Bai, ${ }^{\ddagger}$ Lei Zhang, ${ }^{\dagger}$ Zhongbo Yang, ${ }^{\S}$ Qikui Fan, ${ }^{\dagger}$ Haoquan Zheng, ${ }^{£}$ Yadong Yin, ${ }^{\ddagger}$ and Chuanbo $\mathrm{Gao}^{*, \dagger}$

${ }^{\dagger}$ Center for Materials Chemistry, Frontier Institute of Science and Technology, Xi'an Jiaotong University, Xi'an, Shaanxi 710054, China;

${ }^{\ddagger}$ Department of Chemistry, University of California, Riverside, California 92521, United States;

${ }^{\S}$ Chongqing Key Laboratory of Multi-scale Manufacturing Technology, Chongqing Institute of Green and Intelligent Technology, Chinese Academy of Sciences, Chongqing 400714, China; ${ }^{£}$ Department of Materials and Environmental Chemistry, Stockholm University, Stockholm 10691, Sweden.

Correspondence: gaochuanbo@mail.xjtu.edu.cn

\section{Supporting Video}

Video S1. Electron tomography of a $p$-AuAg NP. The video shows the slices cut out from the reconstructed tomogram, with the cutting position moving from top to bottom of the nanoparticle.

\section{Experimental Section}

Synthesis of Au nanoparticles (NPs) ( 40 nm). Au NPs $(\sim 40 \mathrm{~nm})$ were synthesized by a seeded growth method as described in our earlier reports. ${ }^{[1,2]}$ In a typical synthesis, a seed solution was prepared by injecting $1.2 \mathrm{~mL}$ of ice-cold $\mathrm{NaBH}_{4}(0.1 \mathrm{M})$ into $40 \mathrm{~mL}$ of an aqueous solution containing $0.25 \mathrm{mM}$ trisodium citrate salt (TSC) and $0.25 \mathrm{mM} \mathrm{HAuCl}_{4}$ under vigorous stirring, which was used after $6 \mathrm{~h}$ of ageing. Next, $2 \mathrm{~mL}$ of the seed solution was injected into a growth solution of Au prepared by incorporating $14.4 \mathrm{~mL}$ of poly(vinylpyrrolidone) (PVP, $5 \mathrm{wt} \%$, Mw 10000), $7.2 \mathrm{~mL}$ of ascorbic 
acid $(0.1 \mathrm{M}), 5.4 \mathrm{~mL}$ of $\mathrm{KI}(0.2 \mathrm{M})$, and $1.8 \mathrm{~mL}$ of $\mathrm{HAuCl}_{4}(0.25 \mathrm{M})$ in $72 \mathrm{~mL}$ of $\mathrm{H}_{2} \mathrm{O}$. The resulting $\mathrm{Au}$ NPs were collected by centrifugation, washed with water, and redispersed in $25 \mathrm{~mL}$ of water.

Synthesis of Au@Ag core/shell NPs. In a typical synthesis of Au@Ag core/shell nanospheres (Ag/Au =5), $25 \mathrm{~mL}$ of the Au NPs $(\sim 40 \mathrm{~nm})$ was mixed with $56 \mathrm{~mL}$ of $\mathrm{H}_{2} \mathrm{O}, 144 \mathrm{~mL}$ of PVP $(5 \mathrm{wt} \%$, Mw $10000), 100 \mathrm{~mL}$ of acetonitrile and $20 \mathrm{~mL}$ of ascorbic acid $(0.1 \mathrm{M})$. Then, $22.5 \mathrm{~mL}$ of $\operatorname{AgNO}_{3}(0.1 \mathrm{M})$ was added into the solution at a rate of $1 \mathrm{~mL} / \mathrm{min}$ using a syringe pump. After another $1 \mathrm{~h}, \mathrm{Au} @ \mathrm{Ag}$ NPs were collected by centrifugation and redispersed in $120 \mathrm{~mL}$ of $\mathrm{H}_{2} \mathrm{O}$.

Synthesis of $\mathrm{Au} @ \mathrm{Ag} @ \mathrm{SiO}_{2}$ core/shell NPs. $\mathrm{Au} @ \mathrm{Ag} @ \mathrm{SiO}_{2}$ core/shell NPs were synthesized by a modified Stöber method. Typically, $20 \mathrm{~mL}$ of 16-mercaptohexadecanoic acid ethanolic solution (MHA, $1 \mathrm{mM}$ ) was added dropwise into $60 \mathrm{~mL}$ of the Au@Ag core/shell NPs. To this solution were added in sequence $400 \mathrm{~mL}$ of ethanol, $15 \mathrm{~mL}$ of diethylamine and $2 \mathrm{~mL}$ of tetraethyl orthosilicate under stirring. After 60 min, $\mathrm{Au} @ \mathrm{Ag} @ \mathrm{SiO}_{2}$ core/shell NPs were collected by centrifugation and washed with ethanol and water.

Synthesis of AuAg alloy@SiO 2 core/shell NPs. The Au@Ag@ $\mathrm{SiO}_{2}$ core/shell NPs obtained were dried in vacuum and annealed at $950{ }^{\circ} \mathrm{C}$ in nitrogen for $6 \mathrm{~h}$ for effective alloying of Ag and Au. After that, the nanospheres were sonicated and redispersed in $50 \mathrm{~mL}$ of an alkaline solution containing 5 $\mathrm{mM} \mathrm{NaOH}$. The resulting dispersion was then refluxed at $50{ }^{\circ} \mathrm{C}$ for $6 \mathrm{~h}$, giving rise to AuAgalloy@ $\mathrm{SiO}_{2}$ core/shell NPs with a thin silica shell. The resulting nanoparticles were collected by centrifugation and redispersed in $30 \mathrm{~mL}$ of $\mathrm{H}_{2} \mathrm{O}$.

Synthesis of $p$-AuAg@void@SiO 2 yolk/shell NPs by dealloying. In a typical dealloying process, 2 $\mathrm{mL}$ of the AuAg-alloy@ $\mathrm{SiO}_{2}$ core/shell NPs with thin silica shells was maintained in a water bath at a specific temperature, and $2 \mathrm{~mL}$ of concentrated nitric acid was added under vigorous stirring at a rate of $0.4 \mathrm{~mL} / \mathrm{min}$. After $5 \mathrm{~min}$ of reaction, the resulting $p$-AuAg@void@ $\mathrm{SiO}_{2}$ yolk/shell NPs were collected by centrifugation and redispersed in $2 \mathrm{~mL}$ of $\mathrm{H}_{2} \mathrm{O}$.

Synthesis of $p$-AuAg NPs. $p$-AuAg NPs were obtained by etching of silica from the $p$ AuAg@void@ $\mathrm{SiO}_{2}$ yolk/shell NPs. Typically, 2 mL of $p$-AuAg@void@ $\mathrm{SiO}_{2}$ NPs were dispersed in $10 \mathrm{~mL}$ of an alkaline solution containing $5 \mathrm{mM} \mathrm{NaOH}$ and $0.1 \mathrm{M}$ PVP (by repeating unit, Mw 10,000), and the resulting dispersion was refluxed at $50{ }^{\circ} \mathrm{C}$ for $4 \mathrm{~h}$ to remove the silica shell. The $p$-AuAg NPs were then collected by centrifugation, washed with water and redispersed in $2 \mathrm{~mL}$ of $\mathrm{H}_{2} \mathrm{O}$.

Characterizations. Transmission electron microscopy (TEM) was performed on an FEI Tecnai F20 FEG-TEM operated at an acceleration voltage of $200 \mathrm{kV}$. Scanning electron microscopy (SEM) images and energy dispersive X-ray spectroscopy (EDS) were taken using a field-emission JSM-7000F 
microscope operated at an acceleration voltage of $15 \mathrm{kV}$. Dynamic light scattering (DLS) and $\zeta$ potential analysis were performed on a Beckman Coulter Delsa Nano C particle analyzer. UV-vis spectra $(\lambda: 300-1000 \mathrm{~nm})$ of samples in aqueous solutions were measured on an Ocean Optics HR2000+ES UV-vis-NIR spectrophotometer with a DH-2000-Bal light source. Diffuse reflectance UV-vis spectroscopy ( $\lambda$ : 300-1800 nm) was measured on a SHIMADZU UV-3600 UV-Vis-NIR spectrophotometer equipped with an integrating sphere. The samples were deposited on aminopropyl functionalized glass slide to avoid the significant water absorption band. Fourier transform infrared spectroscopy (FTIR) was performed on a Thermo Fisher Nicolet 6700 FT-IR spectrometer using ATR (Attenuated Total Reflectance) method. Raman spectra were collected using a LabRAM HR800 confocal Raman spectrophotometer equipped with a $633 \mathrm{~nm}$ He-Ne laser.

SERS detection of CV from a silicon substrate. Substrates of $p$-AuAg@void@ $\mathrm{SiO}_{2} \mathrm{NPs}$ (size of $p$ AuAg: $\sim 51 \mathrm{~nm}$, dealloyed at $40^{\circ} \mathrm{C}$ ) were prepared by drying a known quantity of the nanoparticles on a clean silicon wafer $(8 \mathrm{~mm} \times 8 \mathrm{~mm})$. In a typical procedure, $25 \mu \mathrm{L}$ of an aqueous crystal violate $(\mathrm{CV})$ solution $\left(10^{-6} \mathrm{M}\right)$ was dropped and dried on it under lucifugal condition. The substrate was then washed with water for three times. Raman spectra were recorded with a $633 \mathrm{~nm} \mathrm{He}-\mathrm{Ne}$ laser line at room temperature. For all measurements, the size of the laser spot was $0.86 \mu$ m under a $100 \times$ objective, the laser power was $0.3 \mathrm{~mW}$, the power density was $51.67 \mathrm{~kW} \cdot \mathrm{cm}^{-2}$ at the substrate, and the signal acquisition time was fixed to be $2 \mathrm{~s}$. SERS analysis with substrates of $p$-AuAg (size: $\sim 51 \mathrm{~nm}$, dealloyed at $40{ }^{\circ} \mathrm{C}$ ) and $\mathrm{Au}$ NPs (size: $\sim 51 \mathrm{~nm}$ ) were carried out following a similar procedure, with $p$-AuAg and $\mathrm{Au}$ NPs of the same concentration in place of the p-AuAg@void@ $\mathrm{SiO}_{2} \mathrm{NPs}$.

SERS detection of $\mathbf{C V}$ from an aqueous solution. $p$-AuAg@void@ $\mathrm{SiO}_{2}$ (size of $p$-AuAg: $\sim 51 \mathrm{~nm}$, dealloyed at $40^{\circ} \mathrm{C}$ ), $p$-AuAg (size: $\sim 51 \mathrm{~nm}$, dealloyed at $40^{\circ} \mathrm{C}$ ) and Au NPs (size: $\sim 51 \mathrm{~nm}$ ) of the same concentration and volume were incubated with an aqueous solution containing $10^{-6} \mathrm{M} \mathrm{CV}$ for $2 \mathrm{~h}$. The solution was then transferred to a cuvette for the SERS analysis. Raman spectra of the solutions were then recorded with with a $633 \mathrm{~nm} \mathrm{He}-\mathrm{Ne}$ laser line at room temperature. For all measurements, the laser power was $3 \mathrm{~mW}$, and the signal acquisition time was fixed to be $20 \mathrm{~s}$.

Single-particle SERS. $p$-AuAg@void@SiO 2 NPs (size of p-AuAg: 160 nm) or Au NPs (size: 160 $\mathrm{nm})$ were deposited on a clean silicon wafer $(8 \mathrm{~mm} \times 8 \mathrm{~mm})$. Then, $25 \mu \mathrm{L}$ of an aqueous solution of $\mathrm{CV}$ $\left(10^{-6} \mathrm{M}\right)$ was dropped and dried on it under lucifugal condition. The substrate was then washed with water for three times. Raman spectra were recorded from a single nanoparticle on the substrate with a $633 \mathrm{~nm} \mathrm{He}-\mathrm{Ne}$ laser line at room temperature. For both measurements, power density was 51.67 $\mathrm{kW} \cdot \mathrm{cm}^{-2}$, and the signal acquisition time was fixed to be $10 \mathrm{~s}$.

Electron tomography. Electron tomographic studies were performed on the $p$-AuAg NPs dealloyed 
at $0{ }^{\circ} \mathrm{C}$ to characterize the connectivity of the nanopores both within the NPs and to the surface using TEM images. Electron tomography tilt series was collected on JEOL JEM-2100 electron microscope. The tile angle was ranging from $-50^{\circ}$ to $+50^{\circ}$ with an interval of $2^{\circ}$. The sample holder was rotated at a step of $2^{\circ}$ and 51 images were collected totally. The program FIJI ${ }^{[3,4]}$ was used for tilt series alignment, and electron tomographic reconstruction was carried out using the Weighted Back Projection algorithm. The reconstructed 3D tomogram is shown in Video S1.

Finite-difference time-domain (FDTD) simulations. The three-dimensional FDTD simulation was used to calculate the electric field distributions of the $p$-AuAg NPs under different excitations. For simplification, the porous nanoparticles were assumed to be Au. Two models have been applied as shown in Figure S8a to represent porous nanoparticles dealloyed at 40 and $0{ }^{\circ} \mathrm{C}$, respectively. The size of the porous nanospheres was set to be $60 \mathrm{~nm}$. The surrounding medium of the model structure was set to be air. All simulations were performed in Lumerical FDTD Solutions. 


\section{Calculation of the enhancement factor (EF) of a single p-AuAg@void@SiO NP}

The enhancement factor of a single p-AuAg@void@ $\mathrm{SiO}_{2}$ NP (core size: 160 nm) was estimated using the following equation:

$$
\mathrm{EF}=\left(\frac{I_{\text {surface }}}{N_{\text {surface }}}\right) /\left(\frac{I_{\text {solution }}}{N_{\text {solution }}}\right)
$$

where $I_{\text {surface }}$ and $N_{\text {surface }}$ are the Raman intensity probed from a single $p$-AuAg@void@ $@ \mathrm{SiO}_{2} \mathrm{NP}$ and numbers of CV $\left(10^{-6} \mathrm{M}\right)$ molecules absorbed on the single $p$-AuAg@void@ $\mathrm{SiO}_{2} \mathrm{NP}$. $I_{\text {solution }}$ and $N_{\text {solution }}$ are the Raman intensity probed from a CV $\left(10^{-3} \mathrm{M}\right)$ liquid in a capillary and numbers of CV molecules in the focus of the laser beam.

The Raman intensity $I_{\text {surface }}$ was 5045 counts at the peak of $1618 \mathrm{~cm}^{-1}$ (laser power density: 51.67 $\mathrm{kW} \cdot \mathrm{cm}^{-2}$ ). $I_{\text {solution }}$ was measured to be 710 counts (laser power density: $39.8 \mathrm{~kW} \cdot \mathrm{cm}^{-2}$ ), which is equivalent to 922 counts under laser irradiation at $51.7 \mathrm{~kW} \cdot \mathrm{cm}^{-2}$.

$N_{\text {solution }}$ was estimated using the following equation:

$$
N_{\text {solution }}=6.02 \times 10^{23} \mathrm{~mol}^{-1} \times 10^{-3} \mathrm{~mol} / \mathrm{L} \times\left[\pi\left(\frac{d}{2}\right)^{2} \times H\right]=1.8 \times 10^{9}
$$

where $d$ is the diameter of the light spot, $d=\frac{1.22 \lambda}{0.25}=3.1 \mu \mathrm{m} ; \mathrm{H}$ is the effective depth of focus and can be estimated experimentally to be $400 \mu \mathrm{m}$ as follows: ${ }^{[5]}$
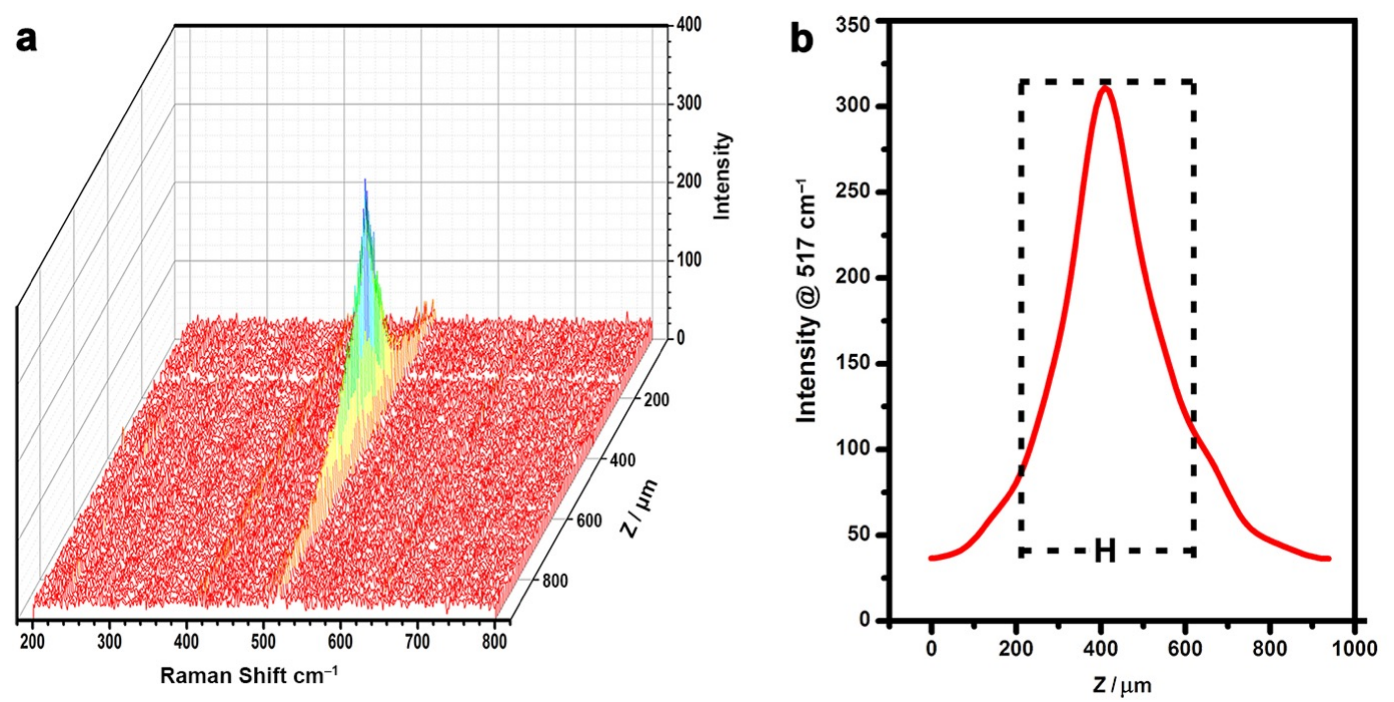

Figure S1. Determination of effective depth of focus. (a) A silicon substrate was put in the laser, and then moved vertically from under focus to over focus. During this process, the Raman signals from 
the silicon substrate were collected. (b) The intensities of the Raman signals were then plotted against the distance the silicon substrate moved, and the effective depth of the focus was then estimated according to this profile.

$N_{\text {surface }}$ can be estimated as:

$$
N_{\text {surface }}=\mathrm{D} \times S_{p}
$$

where $\mathrm{D}$ is the density of CV molecules absorbed on the substrate and the $p$-AuAg@void@ $\mathrm{SiO}_{2} \mathrm{NPs}_{\text {, }}$ which is assumed to be a constant without preferential distribution, and $S_{p}$ is the effective surface area of a single $p$-AuAg@void@ $\mathrm{SiO}_{2} \mathrm{NP}$, and can be estimated by a simplified model (pore size: 10 $\mathrm{nm})$ as $3.7 \times 10^{5} \mathrm{~nm}^{2}$.

In the experiment, $25 \mu \mathrm{L}$ of an aqueous $\mathrm{CV}$ solution $\left(10^{-6} \mathrm{M}\right)$ was dried onto the substrate and dipped 3 times in water to remove weakly absorbed molecules. About $\sim 6.8 \%$ of the $\mathrm{CV}$ molecules remained after dipping, as estimated experimentally (This value is assumed to be independent of the CV concentration, and was determined by UV-vis spectroscopy with CV of a high concentration).

Thus,

$$
\mathrm{N}_{\mathrm{CV}}=25 \times 10^{-6} \mathrm{~L} \times 10^{-6} \mathrm{~mol} / \mathrm{L} \times 6.02 \times 10^{23} \mathrm{~mol}^{-1} \times 6.8 \%=1.0 \times 10^{12}
$$

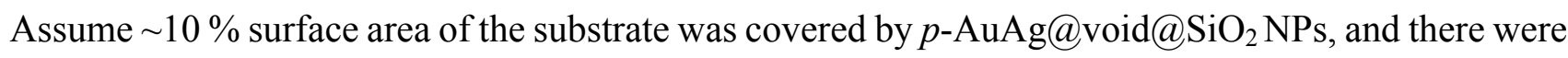
some aggregates of the NPs on the substrate (approximately equivalent to 3 layers of the nanospheres), Thus, $N_{\mathrm{p}}$ (number of $p$-AuAg@void@SiO $\mathrm{Sin}_{2}$ nanospheres on the substrate) can be estimated to be:

$$
N_{p}=3 \times \mathrm{S}_{\mathrm{Si}} \times 10 \% / \pi r^{2}=9.6 \times 10^{8}
$$

where $r$ is the radius of the $p$-AuAg@void@ $\mathrm{SiO}_{2} \mathrm{NPs}, r=80 \mathrm{~nm}, \mathrm{~S}_{\mathrm{Si}}$ is the surface area of the substrate $\left(\mathrm{S}_{\mathrm{Si}}=6.4 \times 10^{13} \mathrm{~nm}^{2}\right)$.

Thus, D can be estimated by the following equation:

$$
\mathrm{D}=\frac{\mathrm{N}_{\mathrm{CV}}}{90 \% \times \mathrm{S}_{\mathrm{Si}}+N_{p} \times \mathrm{S}_{p}}=0.002 \mathrm{~nm}^{-2}
$$

Therefore,

$$
\begin{gathered}
N_{\text {surface }}=\mathrm{D} \times S_{p}=740 . \\
\mathrm{EF}=\left(\frac{I_{\text {surface }}}{N_{\text {surface }}}\right) /\left(\frac{I_{\text {solution }}}{N_{\text {solution }}}\right) \approx 1.3 \times 10^{7}
\end{gathered}
$$




\section{Notes:}

(1) The estimation of the enhancement factors relies on a few assumptions, such as the geometric model of the samples, the amount of the probe molecules and the plasmonic nanoparticles, and the distribution uniformity of both. Therefore, it is difficult to obtain the exact enhancement factor for these plasmonic nanoparticles.

(2) The enhancement factor also varies with the laser wavelength chosen for the SERS analysis. Generally, on-resonance SERS signals are most intense, which gives rise to the largest enhancement factor, as indicated by the FDTD simulation results (Figure 5). In our experiment, the laser wavelength was chosen to be $633 \mathrm{~nm}$, which is far from the LSPR position, and therefore the enhancement factor was much underestimated. For example, the maximal $\mathrm{E}^{4} / \mathrm{E}_{0}^{4}$ can increase by 1200 -fold for the $\mathrm{p}-\mathrm{AuAg}$ NPs when the irradiation wavelength increases from $633 \mathrm{~nm}$ to $818 \mathrm{~nm}$ (on-resonant wavelength in our simulation) (Figure 5). It is thus envisioned that a much favorable enhancement factor could be achieved if a laser with a long wavelength (rather than $633 \mathrm{~nm}$ ) was chosen for the SERS analysis. 


\section{Supporting Data}

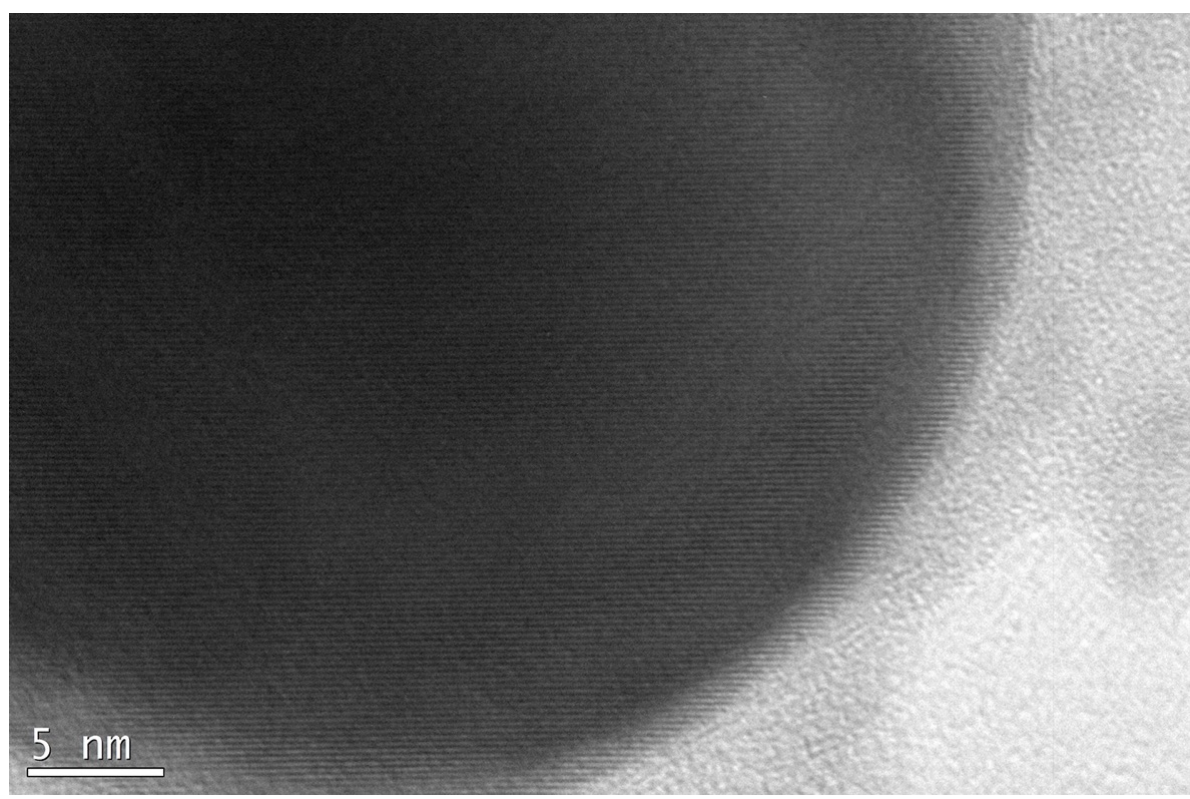

Figure S2. HRTEM image of the AuAg alloy@ $\mathrm{SiO}_{2} \mathrm{NP}$, confirming the single crystallinity of the alloy NP.

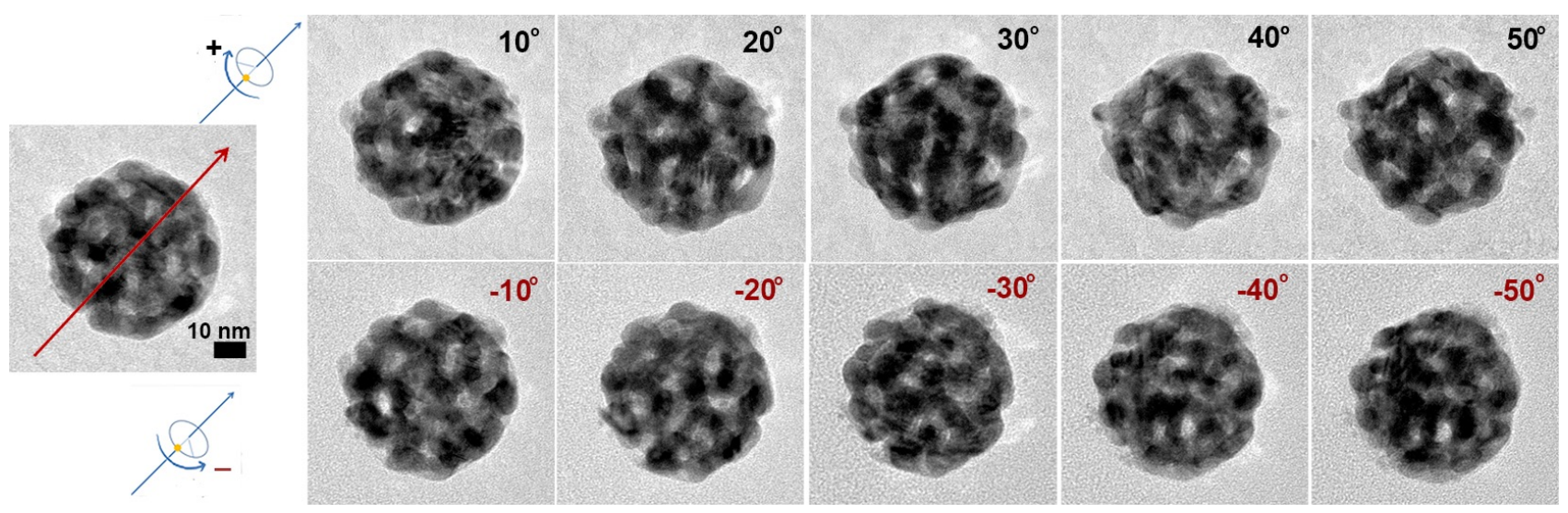

Figure S3. A tilt series of TEM images of an individual $p$-AuAg NP (dealloyed at $0{ }^{\circ} \mathrm{C}$ ) for tomography reconstruction. TEM images were collected with the tilt angles of $-50^{\circ},-40^{\circ},-30^{\circ},-20^{\circ}$, $-10^{\circ}, 0,10^{\circ}, 20^{\circ}, 30^{\circ}, 40^{\circ}, 50^{\circ}$, respectively. 


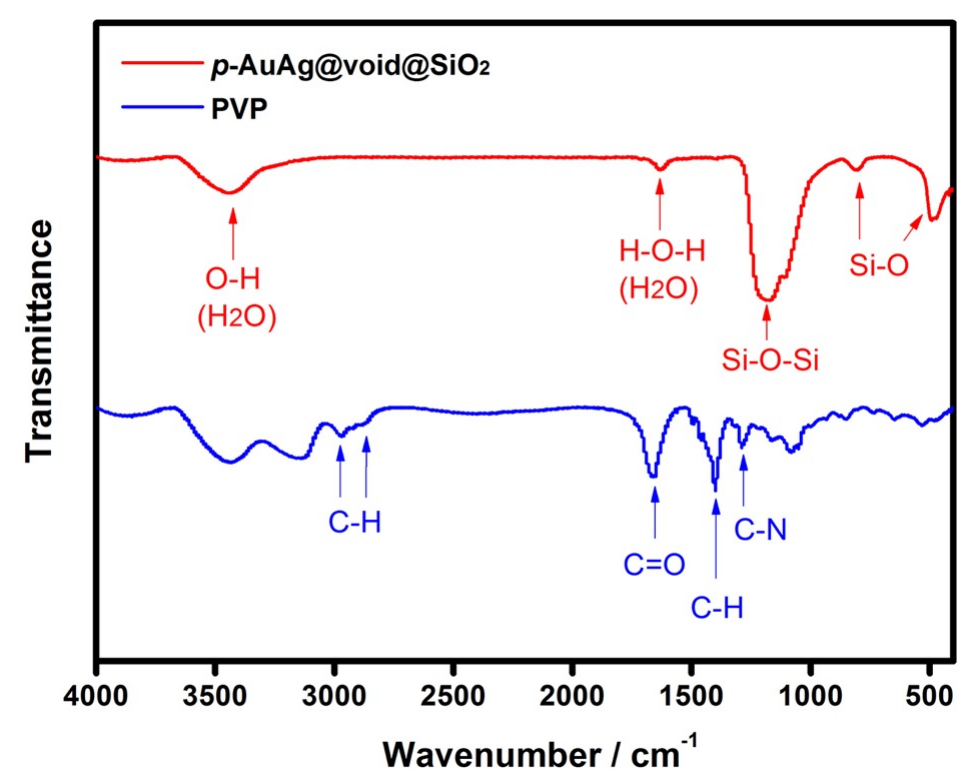

Figure S4. FTIR spectrum of $p$-AuAg@void@ $\mathrm{SiO}_{2}$ yolk/shell NPs. FTIR spectrum of PVP was listed for comparison. No peaks from PVP can be observed from the spectrum of the $p$-AuAg@void@SiO NPs, confirming that PVP as a capping ligand in the synthesis has been completely decomposed, which ensures a clean surface of the $p$-AuAg NPs and thus high accessibility of the hotspots by molecules of interest for sensitive SERS analysis. 


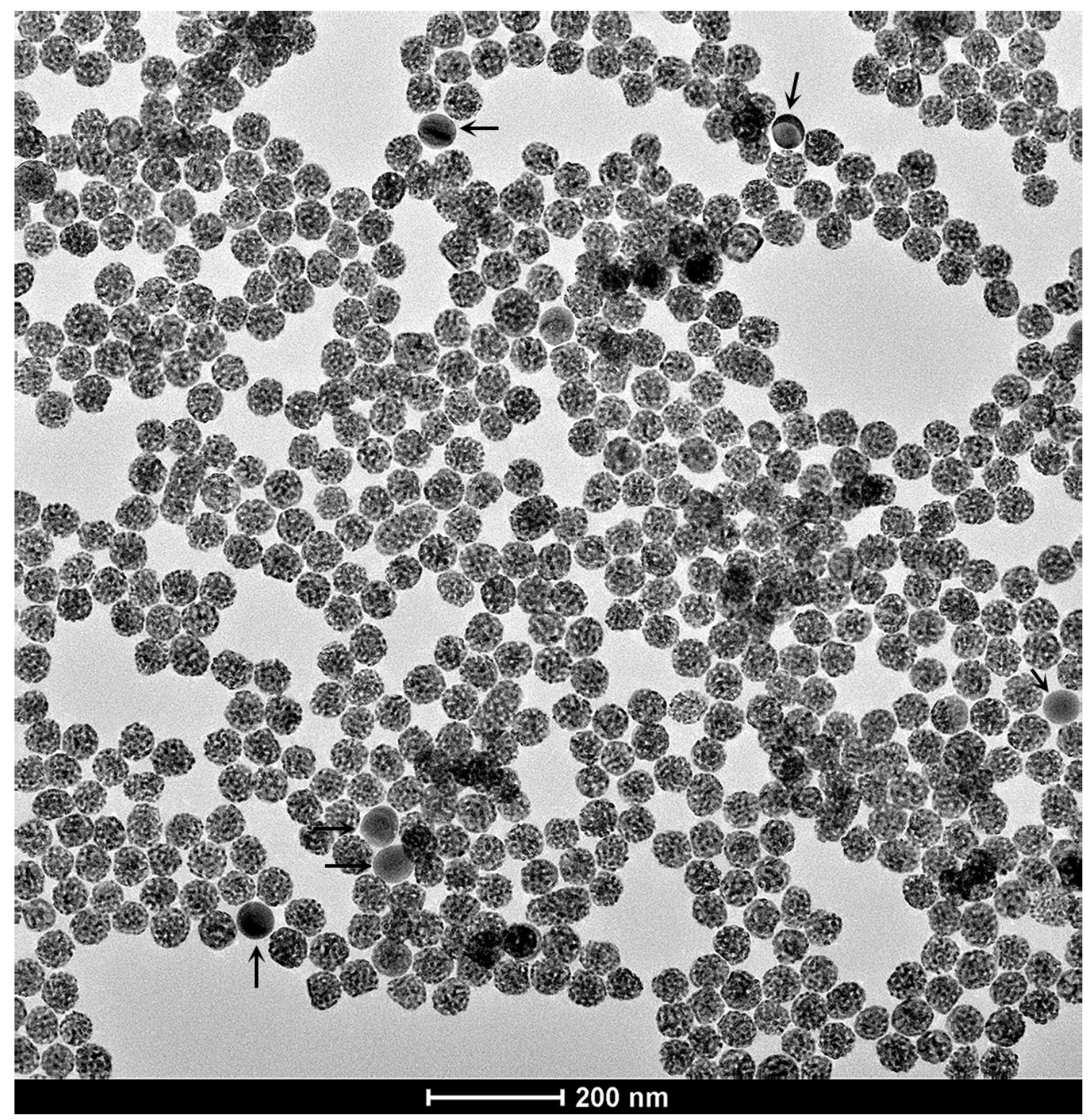

Figure S5. Low-magnification TEM image of the $p$-AuAg NPs obtained by dealloying at $0{ }^{\circ} \mathrm{C}$. Arrows indicate sparse AuAg alloy NPs without being dealloyed. 

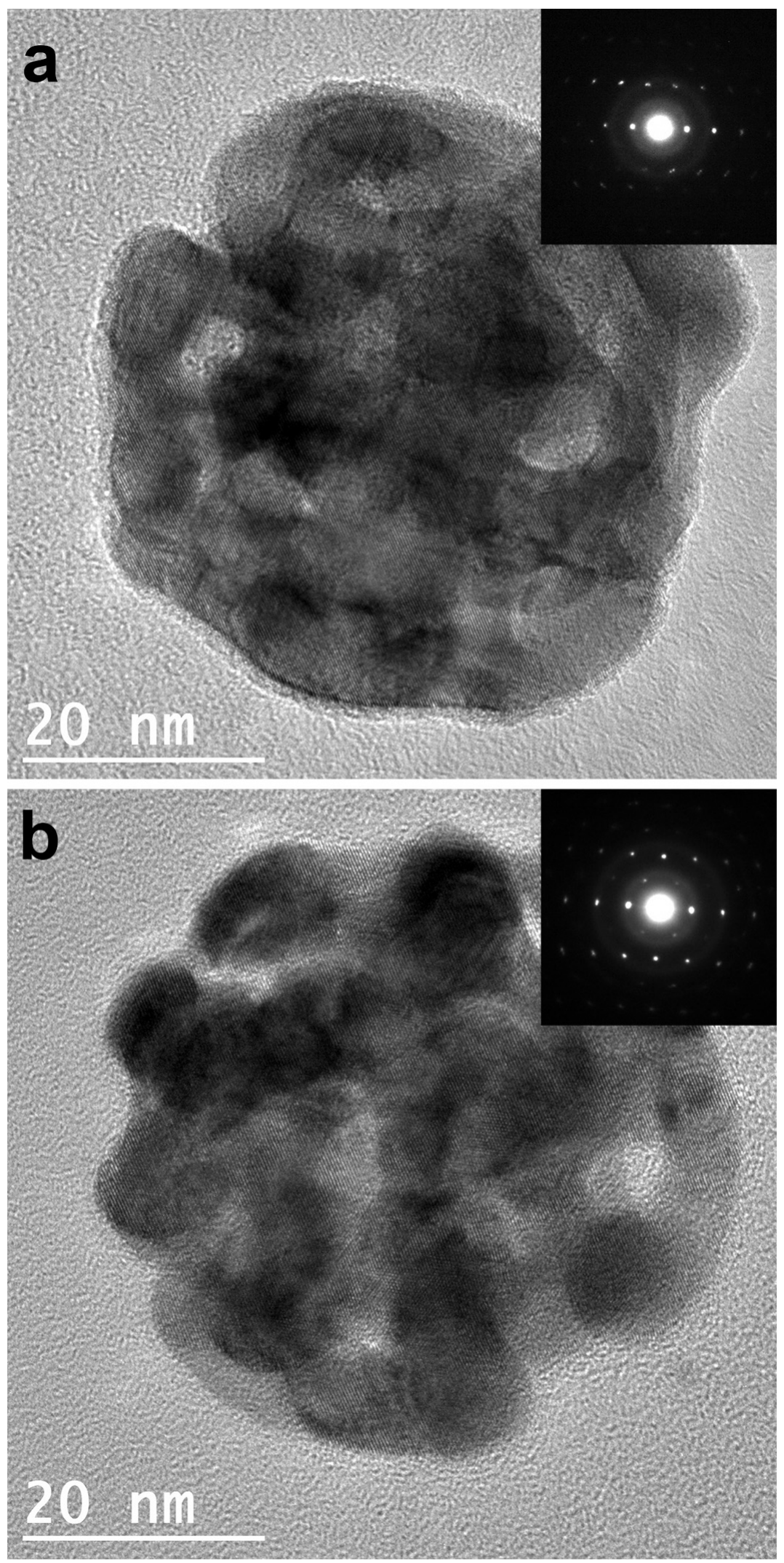

Figure S6. (a-b) HRTEM images of a single $p$-AuAg NP dealloyed at 20 and $40{ }^{\circ} \mathrm{C}$, respectively, showing single crystallinity. Inset: Electron diffractions recorded on a single particle basis. 


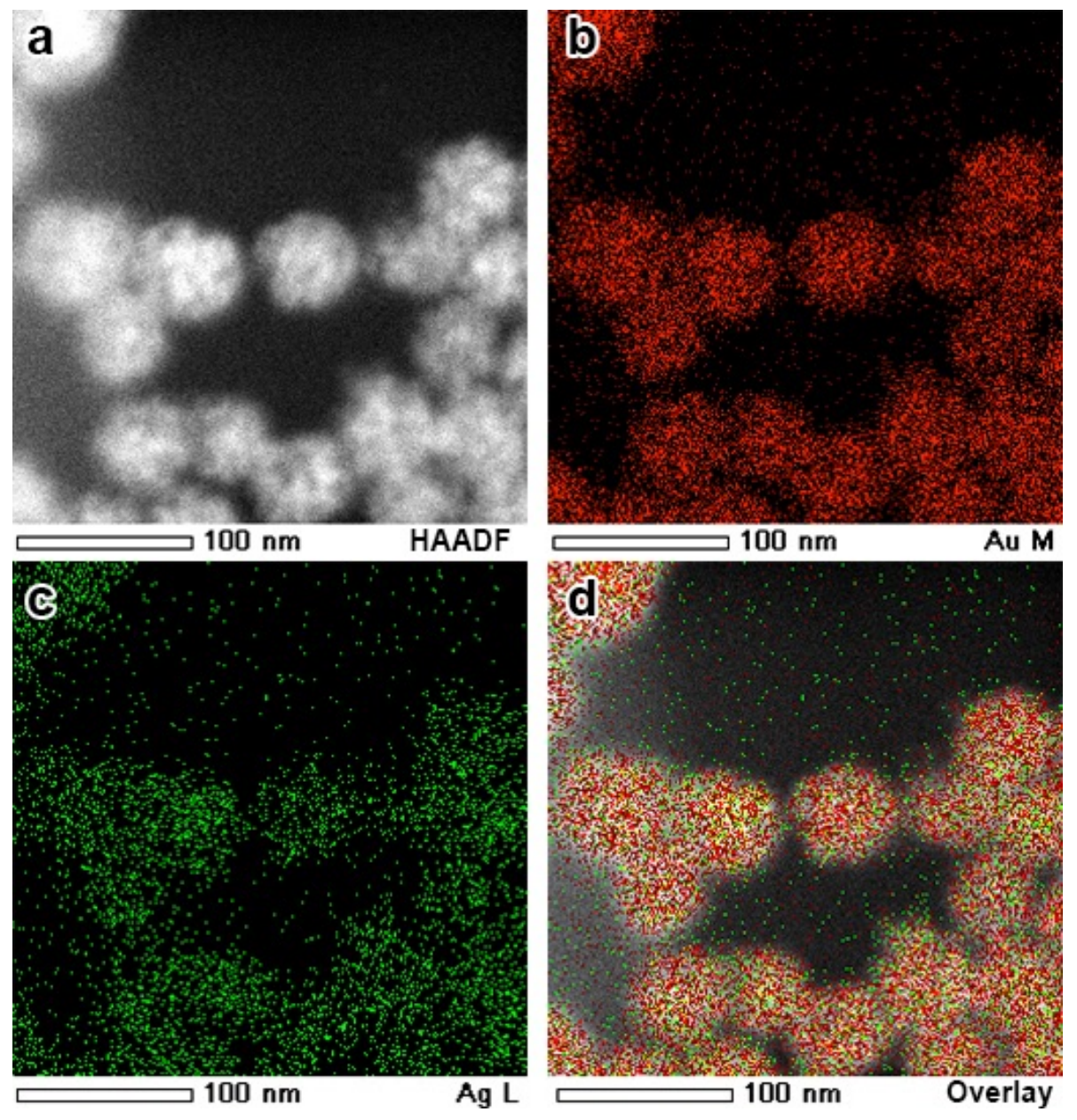

Figure S7. EDS mapping of $p$-AuAg NPs (dealloyed at $0{ }^{\circ} \mathrm{C}$ ). (a) A HAADF-STEM image. (b-c) Distribution of $\mathrm{Au}$ and $\mathrm{Ag}$, respectively. (d) An overlay of the distributions of the Au and Ag elements. 


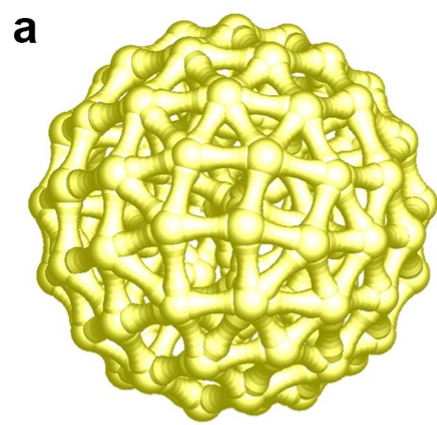

Model 1, with thick ligaments and large nanopaores Size: $60 \mathrm{~nm}$ (Figure 5)

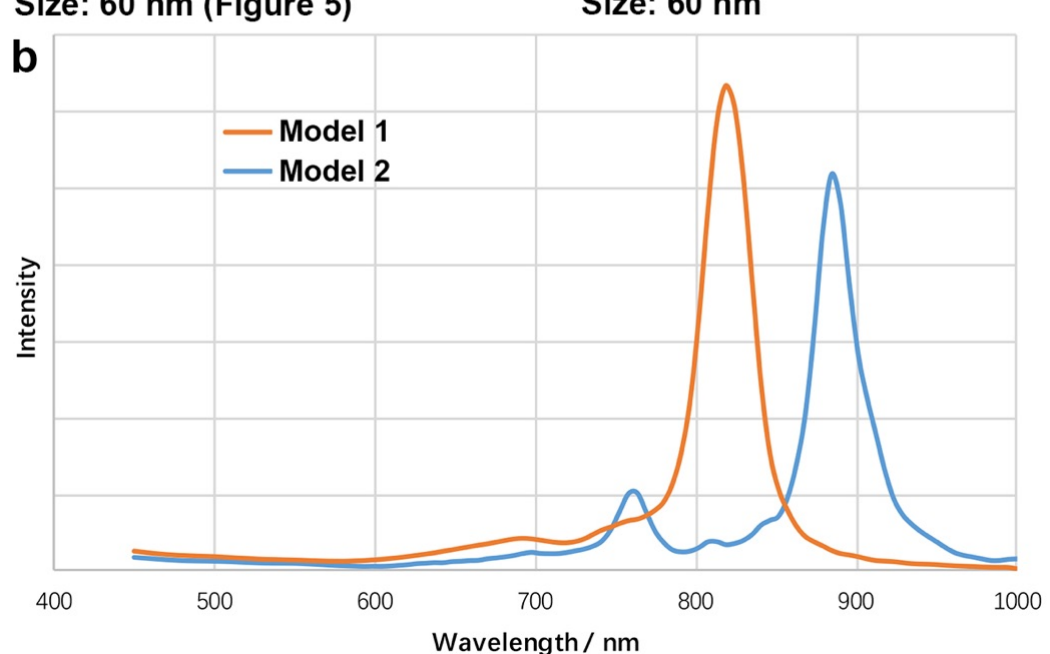

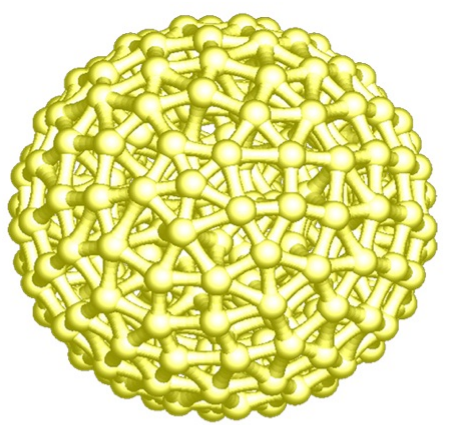

Model 2, with thin ligaments and small nanopores Size: $60 \mathrm{~nm}$

Figure S8. (a) Two models for the FDTD simulations. Model 1 possesses thick ligaments and large nanopores to simulate $p$-AuAg NPs dealloyed at $40{ }^{\circ} \mathrm{C}$, and Model 2 possesses thin ligaments and small nanopores to simulate $p$-AuAg NPs dealloyed at $0{ }^{\circ} \mathrm{C}$. The near-field electromagnetic field distribution of model 1 has been shown in Figure 5. (b) The corresponding extinction spectra calculated by FDTD. 

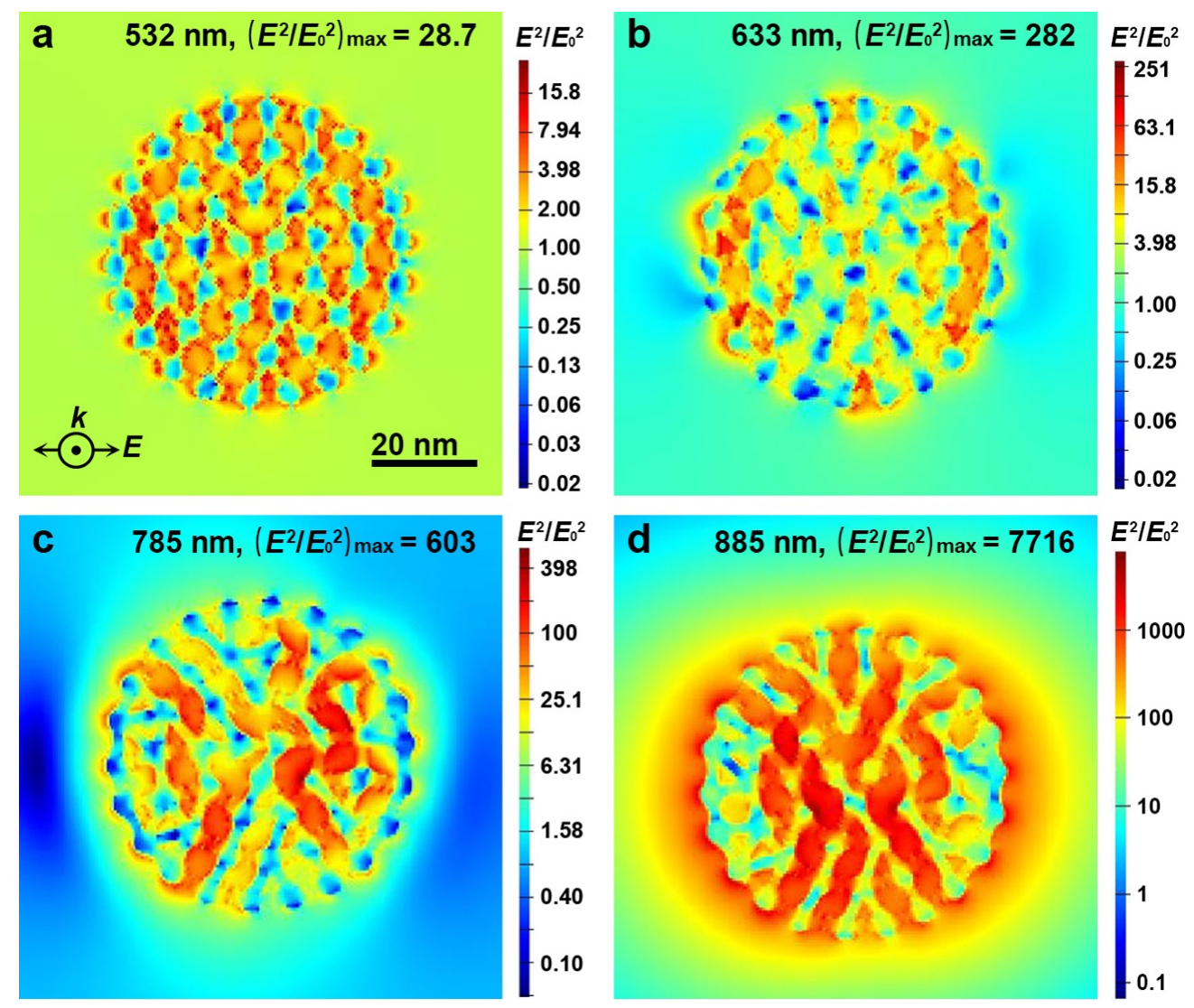

Figure S9. Simulated near-field electric field distributions of the $p$-AuAg NPs with thin ligaments (to simulate $p$-AuAg NPs dealloyed at $0{ }^{\circ} \mathrm{C}$ ), irradiated by a plane wave with wavelength of (a) $532 \mathrm{~nm}$, (b) $633 \mathrm{~nm}$, (c) $785 \mathrm{~nm}$, and (d) $885 \mathrm{~nm}$ (on-resonance condition), respectively. All simulations were performed in Lumerical FDTD Solutions.

Discussion: FDTD simulations reveal that the LSPR of the $p$-AuAg NPs relies on the thickness of the Au-Ag ligaments and the size of the nanopores (Figure S8-S9). The extinction band of the model 2 undergoes a red shift compared with that of model 1, consistent with our spectral observations (Figure 4e-f). It is interesting to note that at short-wavelength irradiations (e.g., 532 and $633 \mathrm{~nm}$ wavelength), the nanopores of both models are excited and produce intense local electromagnetic field that is much close to each other in intensity (Figure 5a-b, S9). 


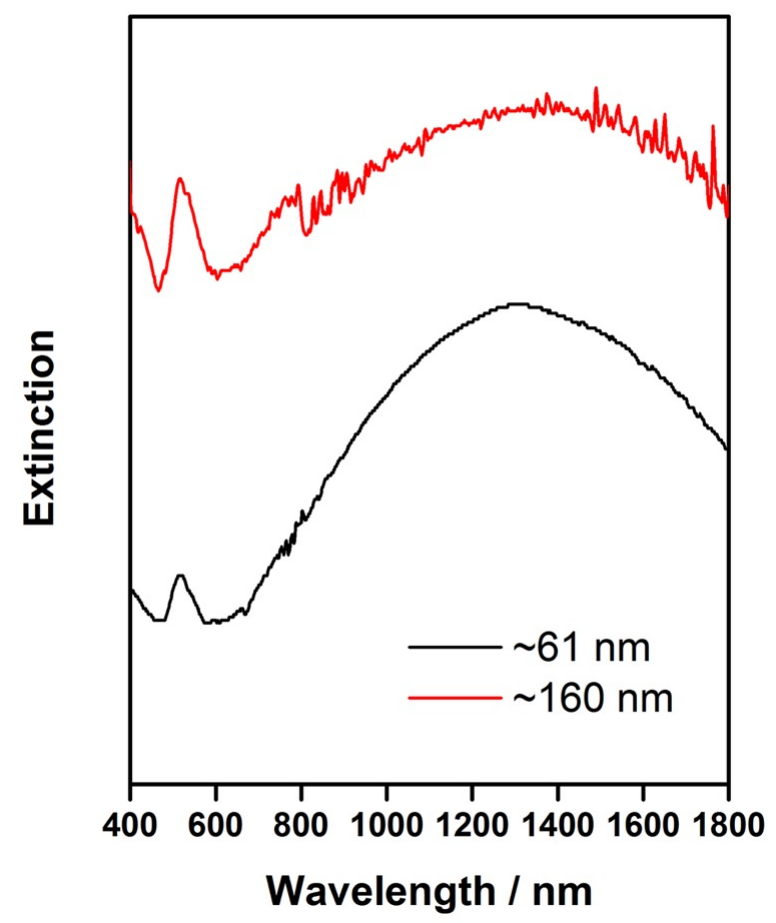

Figure S10. UV-vis-NIR spectra of the p-AuAg@void@ $\mathrm{SiO}_{2}$ yolk/shell NPs with different sizes of the $p$-AuAg NPs. Both materials were dealloyed at $0{ }^{\circ} \mathrm{C}$. 

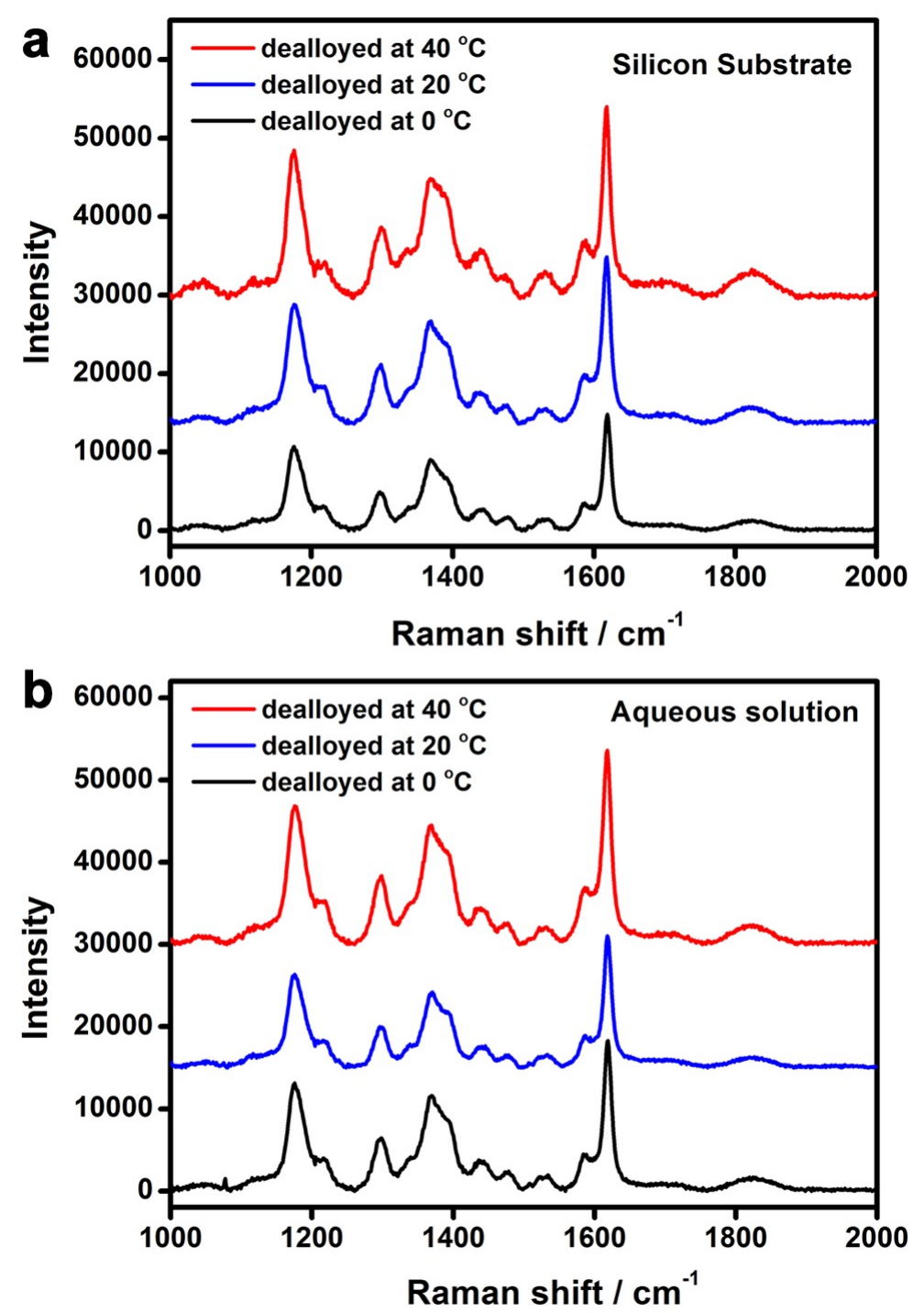

Figure S11. SERS activities of the p-AuAg@void@ $\mathrm{SiO}_{2}$ NPs obtained by dealloying at different temperatures. (a) Raman signals of $\mathrm{CV}\left(10^{-6} \mathrm{M}\right)$ recorded from silicon substrates deposited with the respective nanoparticles. Laser wavelength: $633 \mathrm{~nm}$; power density: $51.67 \mathrm{~kW} \cdot \mathrm{cm}^{-2}$, acquisition time: $2 \mathrm{~s}$. (b) Raman signals recorded from aqueous solutions of $\mathrm{CV}\left(10^{-6} \mathrm{M}\right)$ with different suspensions of the nanoparticles. Laser wavelength: $633 \mathrm{~nm}$; power density: $39.8 \mathrm{~kW} \cdot \mathrm{cm}^{-2}$, acquisition time: $20 \mathrm{~s}$. As predicted by the FDTD simulations (Figure 5, S9), $p$-AuAg NPs with thick and thin ligaments produce intense and almost identical (in terms of maximal $\mathrm{E}^{2} / \mathrm{E}_{0}^{2}$ ) electromagnetic field in the nanopores, which explains the similar SERS activities using these porous nanoparticles. 

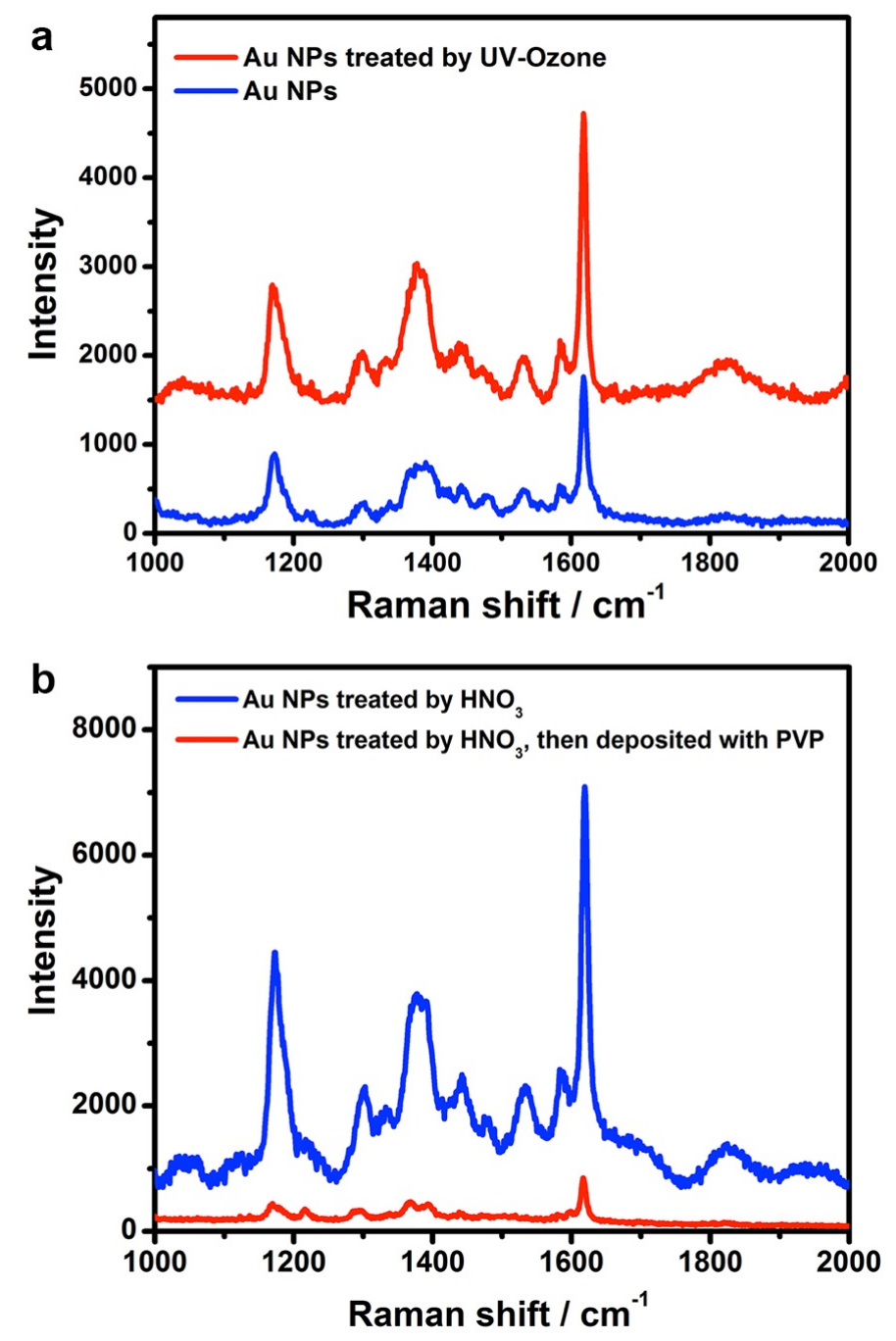

Figure S12. Effect of capping ligands on the SERS activities of the nanoparticles, exemplified by PVP-capped Au NPs. (a) A known concentration and volume of the Au NPs were deposited on a silicon substrate, with PVP removed by UV ozone. CV $\left(10^{-6} \mathrm{M}\right)$ was then dried on the substrate for SERS analysis (Laser power density: $51.67 \mathrm{~kW} \cdot \mathrm{cm}^{-2}$, acquisition time: $2 \mathrm{~s}$ ). A significant increase in the intensity of the Raman signals was observed compared with the Raman signals from the substrate of the as-synthesized Au NPs. (b) The Au NPs were washed with $\mathrm{HNO}_{3}$ to remove the capping agent PVP, which were then deposited on a silicon substrate. CV $\left(10^{-6} \mathrm{M}\right)$ was then dried on the substrate for SERS analysis (Laser power density: $51.67 \mathrm{~kW} \cdot \mathrm{cm}^{-2}$, acquisition time: $2 \mathrm{~s}$ ). Strong Raman signals can be observed from the substrate. However, if a low concentration of PVP was dried on the substrate before depositing of $\mathrm{CV}$, the resulting Raman signals become significantly decreased. 
Table S1. Typical enhancement factors (EFs)of plasmonic nanocrystals with interior nanogaps or shape tips. (In order of decreasing EF)

\begin{tabular}{cccc}
\hline Entry & EF & $\begin{array}{r}\text { Excitation } \\
\text { wavelength }\end{array}$ & Reference \\
\hline Star-like gold nanoparticles (on Au film) & & & \\
\hline
\end{tabular}

Au nanostars (on Au substrate)

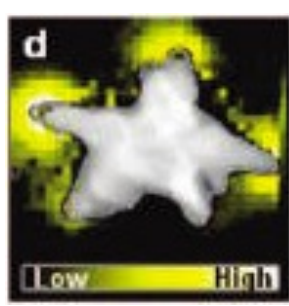

$\sim 10^{10} \quad 785 \mathrm{~nm}$

$[7]$

Wrinkled nanoporous gold film

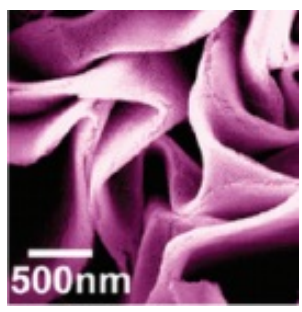
$\sim 10^{9}$
$633 \mathrm{~nm}$

[8]

Au-Ag Alloy nanourchins

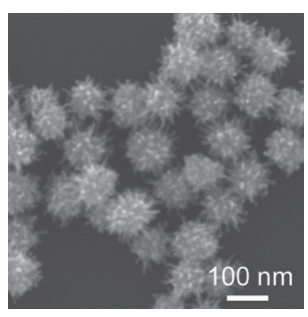
$\sim 10^{9}$
$633 \mathrm{~nm}$

[9]

Gold nanostars (on Au film)

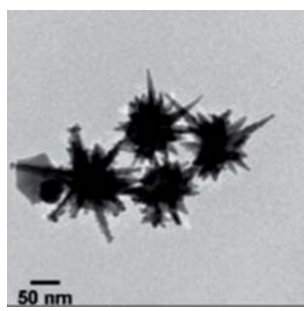

$\sim 10^{9} \quad 633 \mathrm{~nm}$

[10] 
Au nanoparticles with 1-nm interior gap

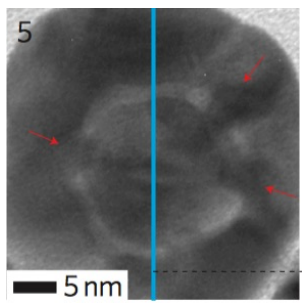

$$
1 \times 10^{8}-5 \times 10^{9} \quad 633 \mathrm{~nm}
$$

Nanoporous gold disk

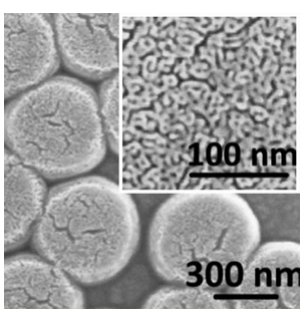

$$
\sim 10^{8} \quad 785 \mathrm{~nm}
$$

p-AuAg@void@ $\mathrm{SiO}_{2}$

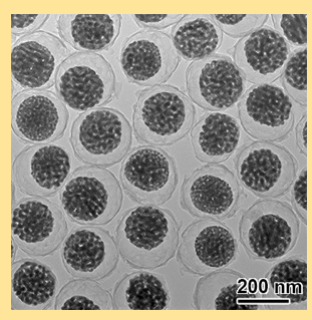

$$
\sim 1.3 \times 10^{7} \quad \begin{gathered}
633 \mathrm{~nm} \\
\text { (off-resonant) }
\end{gathered}
$$

This work

Mesoscopic Au meatball particles

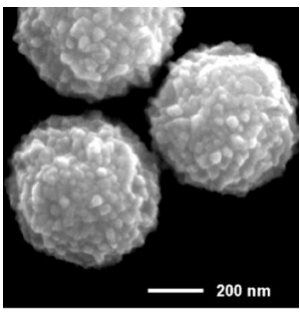

$$
10^{7} \sim 10^{8} \quad 785 \mathrm{~nm}
$$

Gold nanostars

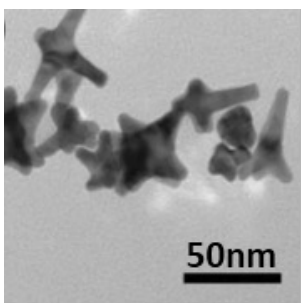

$$
\sim 10^{7} \quad 785 \mathrm{~nm}
$$

Porous gold nanoparticles

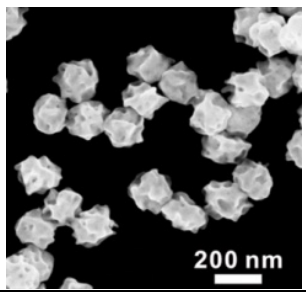

$\sim 10^{6} \quad 785 \mathrm{~nm}$


Coarse Au nanoparticles

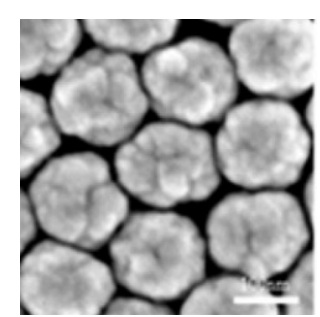

$\sim 10^{6} \quad 647 \mathrm{~nm}$

[16]

Porous AuAg alloy nanoparticles

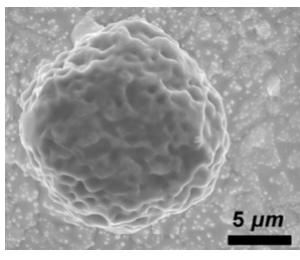

$\sim 10^{5} \quad$ N.A.

[17]

Self-assembled plasmonic vesicles

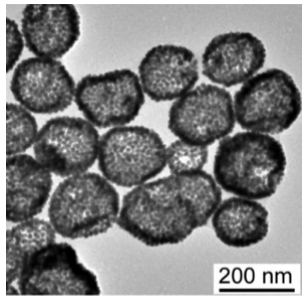

$7.2 \times 10^{4} \quad 633 \mathrm{~nm}$

Nanoporous gold nanoframes

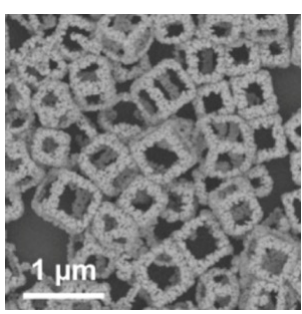

$\sim 10^{4} \quad 785 \mathrm{~nm}$

[19]

Anisotropically etched silver

nanoparticles

$3 \times 10^{4}-5 \times 10^{4} \quad \begin{array}{cc}514,633,785 \\ \mathrm{~nm}\end{array}$

Pd@AuCu core-shell planar tetrapods

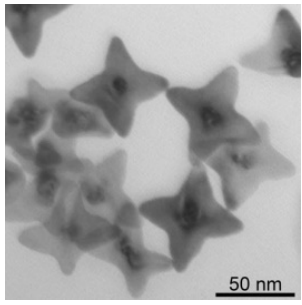

$\sim 9.0 \times 10^{3} \quad 514 \mathrm{~nm}$

[21] 


\section{References:}

1. Gao, C.; Goebl, J.; Yin, Y. J. Mater. Chem. C 2013, 1, 3898-3909.

2. Gao, C.; Vuong, J.; Zhang, Q.; Liu, Y.; Yin, Y. Nanoscale 2012, 4, 2875-2878.

3. Schindelin, J.; Arganda-Carreras, I.; Frise, E.; Kaynig, V.; Longair, M.; Pietzsch, T.; Preibisch, S.; Rueden, C.; Saalfeld, S.; Schmid, B.; Tinevez, J.-Y.; White, D. J.; Hartenstein, V.; Eliceiri, K.; Tomancak, P.; Cardona, A. Nat. Meth. 2012, 9, 676-682.

4. Schneider, C. A.; Rasband, W. S.; Eliceiri, K. W. Nat. Meth. 2012, 9, 671-675.

5. Cai, W. B.; Ren, B.; Li, X. Q.; She, C. X.; Liu, F. M.; Cai, X. W.; Tian, Z. Q. Surf. Sci. 1998, 406, 9-22.

6. Perez-Mayen, L.; Oliva, J.; Torres-Castro, A.; De la Rosa, E. Nanoscale 2015, 7, 10249-10258.

7. Rodríguez-Lorenzo, L.; Álvarez-Puebla, R. A.; Pastoriza-Santos, I.; Mazzucco, S.; Stéphan, O.; Kociak, M.; Liz-Marzán, L. M.; García de Abajo, F. J. J. Am. Chem. Soc. 2009, 131, 4616-4618.

8. Zhang, L.; Lang, X.; Hirata, A.; Chen, M. ACS Nano 2011, 5, 4407-4413.

9. Liu, Z.; Yang, Z.; Peng, B.; Cao, C.; Zhang, C.; You, H.; Xiong, Q.; Li, Z.; Fang, J. Adv. Mater. 2014, 26, 2431-2439.

10. Indrasekara, A. S.; Meyers, S.; Shubeita, S.; Feldman, L. C.; Gustafsson, T.; Fabris, L. Nanoscale 2014, 6, 8891-8899.

11. Lim, D. K.; Jeon, K. S.; Hwang, J. H.; Kim, H.; Kwon, S.; Suh, Y. D.; Nam, J. M. Nat. Nanotech. 2011, 6, 452-460.

12. Qi, J.; Motwani, P.; Gheewala, M.; Brennan, C.; Wolfe, J. C.; Shih, W. C. Nanoscale 2013, 5, 4105-4109.

13. Wang, H.; Halas, N. J. Adv. Mater. 2008, 20, 820-825.

14. Saverot, S.; Geng, X.; Leng, W.; Vikesland, P. J.; Grove, T. Z.; Bickford, L. R. RSC Adv. 2016, 6, 29669-29673.

15. Zhang, Q.; Large, N.; Nordlander, P.; Wang, H. J. Phys. Chem. Lett. 2014, 5, 370-374.

16. Zhang, Y.; Wang, L.-M.; Tan, E.-Z.; Yang, S.-H.; Li, L.-D.; Guo, L. Chin. Chem. Lett. 2015, 26, $1426-1430$.

17. Cao, Q.; Yuan, K.; Liu, Q.; Liang, C.; Wang, X.; Cheng, Y. F.; Li, Q.; Wang, M.; Che, R. ACS Appl. Mater. Inter. 2015, 7, 18491-18500.

18. Song, J.; Zhou, J.; Duan, H. J. Am. Chem. Soc. 2012, 134, 13458-13469.

19. Chew, W. S.; Pedireddy, S.; Lee, Y. H.; Tjiu, W. W.; Liu, Y.; Yang, Z.; Ling, X. Y. Chem. Mater. 2015, 27, 7827-7834.

20. Mulvihill, M. J.; Ling, X. Y.; Henzie, J.; Yang, P. J. Am. Chem. Soc. 2009, 132, 268-274.

21. Meng, M.; Fang, Z.; Zhang, C.; Su, H.; He, R.; Zhang, R.; Li, H.; Li, Z. Y.; Wu, X.; Ma, C.; Zeng, J. Nano Lett. 2016, 16, 3036-3041. 